\title{
Low Cost Obstacle Avoidance Robot with Logic Gates and Gate Delay Calculations
}

\author{
Dewan Mohammed Abdul Ahad ${ }^{1}$, Dewan Mohammed Rashid ${ }^{2}$, Md. Sajid Hossain ${ }^{3, *}$ \\ ${ }^{1}$ Electrical \& Electronic Engineering, Atish Dipankar University of Science \& Technology, Dhaka, Bangladesh \\ ${ }^{2}$ Electrical \& Electronic Engineering, Ahsanullah University of Science \& Technology, Dhaka, Bangladesh \\ ${ }^{3}$ Electrical \& Electronic Engineering, American International University-Bangladesh, Dhaka, Bangladesh \\ Email address: \\ ahad_dewan@yahoo.com (D. M. A. Ahad), rashid.udit@gmail.com (D. M. Rashid), sajidh@outlook.com (Md. S. Hossain) \\ ${ }^{*}$ Corresponding author
}

\section{To cite this article:}

Dewan Mohammed Abdul Ahad, Dewan Mohammed Rashid, Md. Sajid Hossain. Low Cost Obstacle Avoidance Robot with Logic Gates and Gate Delay Calculations. Automation, Control and Intelligent Systems. Vol. 6, No. 1, 2018, pp. 1-7. doi: 10.11648/j.acis.20180601.11

Received: November 8, 2017; Accepted: January 16, 2018; Published: February 6, 2018

\begin{abstract}
As a fast growing field, robots are greatly used to achieve the desired task more accurately and mitigate the difficulties in odd environments where human face immense difficulties. In this paper an obstacle avoidance robot has been designed using basic gates. It can detect the obstacle and directs itself with the help of five sensors. When sensor detects an obstacle it gives the pulse high and vice-versa. A differential drive model has been chosen, which has two wheels and a cluster wheel. Left and right motor are used as a physical machine and it will be controlled by logic; K-map has been used to do it. Basic gates help to execute the equation of motors as well as to make robot faster, precise and efficient. To make more comprehensible comparative time delay estimation has been added in this paper.
\end{abstract}

Keywords: DDMR, Logic Gate, IR Sensor, K-Map, Gate Delay

\section{Introduction}

Robotics is a mystery. More it becomes mystery more it relates to our life. Nowadays, indoor or outdoor wherever people goes robot follows. Means, it assist in everywhere where people needs. Now, to follow someone robot needs a special kind of behaviors [1] that give it the ability to avoid an obstacle and reach its goal [2]-[3]. Avoid obstacle robot can be humanoid or can be mobile. This paper concentrates on mobile obstacle avoidance robot.

The robot that moves from one spot to another is a mobile robot [4]. In precise the robot which has the mobility (air, land, underwater), a certain level of autonomy and perception ability is called mobile robot [5]. To avoid an obstacle is the primary goal for any smart mobile robot. When the robot is going to accomplish a goal-seeking task, the whole task divided into chunk. Obstacle avoidance is the initial one [6]. Obstacle avoidance is the fundamental concern of an outstanding mobile robot. Collusion avoidance algorithm and ranging sensor features helps the robot to detect the obstacle and turn or stop at certain time [7].
Modeled obstacle avoidance robot is a differential drive robot model with five IR sensors arranged in five sides. The robot runs with 12 volt batteries, chassis and logic gates. The time delay calculations of the gates have been studied which ensure the robot's authenticity.

\section{Design Approach}

Design an obstacle avoidance robot not only requires a combination of hardware and software but also an environmental situation. Two facts have to consider at the time of designing a robot. The first is the world is fundamentally unknown place. The robot does not know the where the obstacles are, when it driving in to it's location. So, there are no ways to plan in advance about the robot's perspectives. Next, obstacles move around. A man can pass by the robot. So, avoid-obstacles means robot would not crush into things [1]. For that the IR sensors have been used. Sensor will inform the robot what the world around.

Now, these sensors give the instruction to the logic gates. Logic gates are the brain of the robot. According to their 
instructions, wheels spines right or left.

Finally, a robot model has been chosen. In order to design a robot, a robot model is needed and differential drive robot model is the easiest and common model to study, which is based on equation 11 and equation 12. A differential drive robot has two wheels and a caster wheel on back [4]. When left motor spin and right motor stop then robot moves left. Again, when right motor spin and left motor stop then robot move right. When both motor spin simultaneously, then robot goes straight [8]. Every element has a coordinate. So, designed robot needs a coordinate. In this case $\mathrm{x}, \mathrm{y}$ and $\theta$ is the position and orientation of the obstacle avoidance robot respectively. After transition between inputs and states following equations have been obtained [8]-[9].

$$
\begin{gathered}
\dot{x}=\frac{R}{2}\left(V_{r}+V_{l}\right) \cos \theta, \\
\dot{y}=\frac{R}{2}\left(V_{r}+V_{l}\right) \sin \theta, \\
\dot{\theta}=\frac{R}{2}\left(V_{r}-V_{l}\right),
\end{gathered}
$$

These equations are the differential drive robot model equations, where, $\frac{x}{x} \cdot \vec{y}$ and $\cdot \dot{\theta}$ is the process of $\mathrm{x}$ position, $\mathrm{y}$ position of the robot change and the process of robot turning respectively. So, this is a model in terms of mapping control on to states, "Based on the limitations of equations (1), (2) and (3) [9], unicycle model has been used", which successfully overcome the wheel velocities problem. Then, translational velocity $(\mathrm{v})$, that is speed and angular velocity $(\omega)$ has been considered instead of considering wheel velocities. So, $\mathrm{v}$ and $\omega$ are two new input. Now, to map them a second order unicycle dynamics is needed, they are

$$
\begin{gathered}
\dot{x}=v \cos \theta, \\
\dot{y}=v \sin \theta, \\
\dot{\theta}=\omega,
\end{gathered}
$$

But, "Equations (4), (5) and (6) are not the differential drive model", $v$ and $\omega$ of the equation (4), (5) and (6) are the control input but $\mathrm{V}_{\mathrm{r}}$ and $\mathrm{V}_{1}$ of equation (1) are actual control parameters. "Both the control parameter has been mapped to obtain the following equations [1], [8]

$$
\begin{gathered}
v=\frac{R}{2}\left(v_{r}+v_{l}\right), \\
\frac{2 v}{R}=v_{r}+v_{l},
\end{gathered}
$$

$$
\begin{aligned}
& \omega=\frac{R}{L}\left(v_{r}-v_{l}\right), \\
& \frac{\omega L}{R}=v_{r}-v_{l},
\end{aligned}
$$

Here, equation (8) connects the translational velocity to the real velocities. Equation (8) and (10) are linear equations". This has been solved to find the desired robot model.

$$
\begin{aligned}
& v_{r}=\frac{2 v+\omega L}{2 R}, \\
& v_{l}=\frac{2 v-\omega L}{2 R},
\end{aligned}
$$

So, equation (11) and equation (12) is the desired robot model, where, L (length of the wheel base, means how far away the wheel from each other) is wheel base and $\mathrm{R}$ is the radius that says about the size of the wheel, $\mathrm{V}_{\mathrm{r}}$ is the rate of right wheel turning and $\mathrm{v}_{1}$ is the rate of left wheel turning, $\mathrm{v}$ and $\omega$ are translational velocity and angular velocity respectively [1]. Figure 1 shows the differential drive robot model.

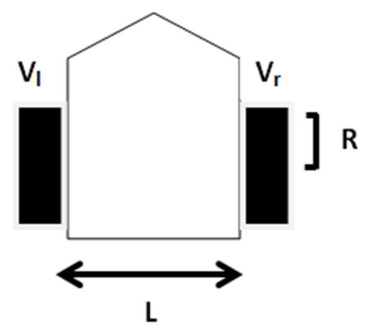

Figure 1. Differential Drive Robot Model.

Designing and implementation of an obstacle avoidance robot is placed not only the robot model or sensor moreover characteristics of the motors, driver IC, circuit diagram and most importantly selection of the gates. Three basic steps give a complete figure of the robot as step-1 was circuit simulation, step-2 was hardware setup and step-3 was circuit implementation. Each component has been studied in detail and has been tested. The block diagram of working principle is given in Figure 2.

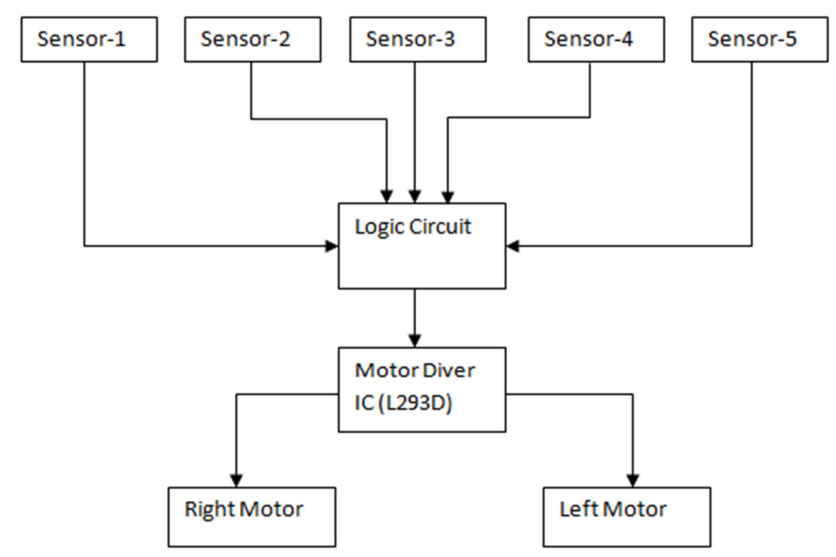

Figure 2. Block diagram for implemented obstacle avoidance robot. 


\section{Carry out Equation}

Digital logic only recognizes 0 and 1 . When the robot faces obstacles then IR sensors sense logic 1 or vice versa. Based on five sensors different scenarios have been created. Two motor has been used, named LM (left motor) and RM (right motor). Similarly, FS (front sensor), LLS (lower left sensor), LRS (lower right sensor), LCS (left corner sensor), RCS (right corner sensor).

A. Scenario-1 (Move Forward) [LM=1, RM=1]

When robot does not sense any object, DDMR moves forward. Obstacles at left side, right side and the both corner, DDMR also moves forward. Again, when both the lowerright and lower-left and front is clear but have obstacle in the both left-right corner then robot moves forward. Scenario1 has been shown in Figure 3.

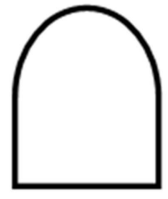

(a)

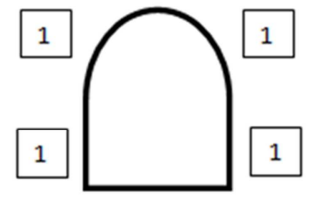

(b)

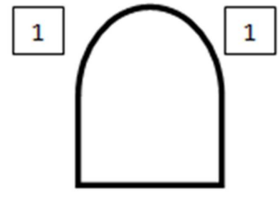

(c)
Figure 3. (a) All side are free from obstacle, (b) Just front side is free, (c) lower right-left and front sides are free from obstacle.

B. Scenario-2 (Move Right) [LM=0, RM=1]

When lower right side is free and rest of the sides are full of obstacle then the robot goes right. If the right corner side is free and other four sensor sense obstacle robot also goes to right direction. Figure 4 shows better illustration.

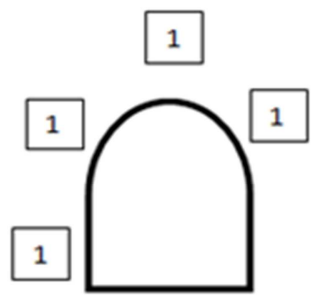

(a)

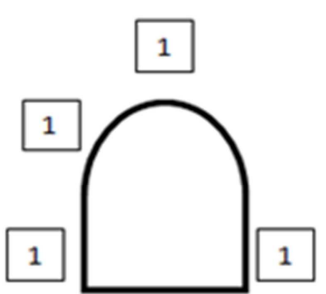

(b)
Figure 4. (a) Lower right side is free; (b) Upper right corner is free.

C. Secnario-3 (Move Left Side) [LM=1, RM=0]

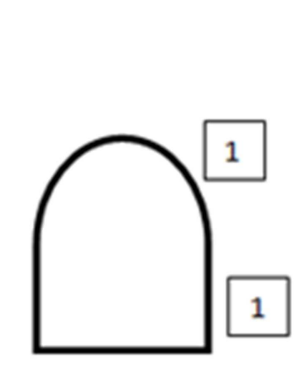

(a)

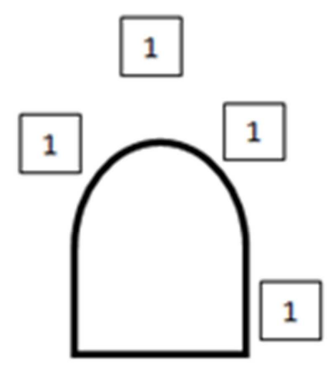

(b)
Figure 5. (a) Left and front side is free from obstacle; (b) Lower left side is free from obstacle.
When left side and front side are free but right side is full of obstacle, robot goes to left. Again, when lower left side is free but there are obstacles in left corner side, front side, right side and right corner side, then robot also goes to left direction that have been shown in Figure 5.

D. Secnario-4 (Special Case) $[\mathrm{LM}=0, \mathrm{RM}=0]$

When DDMR senses obstacle in all his side, robot stops. When, lower left side and lower right side is free but there are obstacles in right corner side, left corner side and front side, robot stops moving. This scenario has been shown in Figure 6.

From the table 1, K-map has been drawn and from the Kmap left motor and right motor equation has been obtained.

$$
\begin{aligned}
& \mathrm{LM}=\mathrm{A}^{\prime} \mathrm{B}^{\prime} \mathrm{CE}+\mathrm{A}^{\prime} \mathrm{B}^{\prime} \mathrm{CD}+\mathrm{A}^{\prime} \mathrm{CDE}+\mathrm{A}^{\prime} \mathrm{B}^{\prime} \mathrm{C}^{\prime} \mathrm{D}^{\prime} \mathrm{E}^{\prime}+\mathrm{ABCDE} \\
& \mathrm{RM}=\mathrm{A}^{\prime} \mathrm{B}^{\prime} \mathrm{D}^{\prime} \mathrm{E}+\mathrm{A}^{\prime} \mathrm{B}^{\prime} \mathrm{CE}^{\prime}+\mathrm{AB}^{\prime} \mathrm{CE}+\mathrm{AB}^{\prime} \mathrm{C}^{\prime} \mathrm{DE}^{\prime}+\mathrm{ABCDE}
\end{aligned}
$$

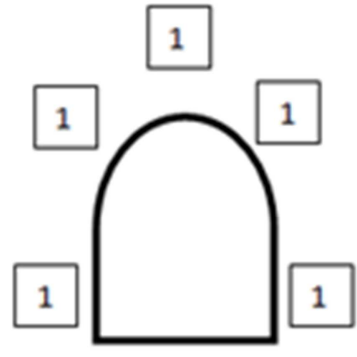

(a)

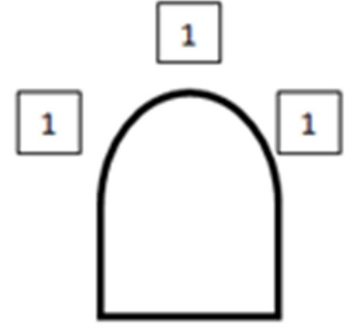

(b)
Figure 6. (a) DDMR stops (b) Lower left \& right are free.

Table 1. Truth Table.

\begin{tabular}{llllllll}
\hline No & LS & RS & LCS & RCS & FS & LM & RM \\
\hline 1 & 0 & 0 & 0 & 0 & 0 & 1 & 1 \\
2 & 1 & 1 & 1 & 1 & 0 & 1 & 1 \\
3 & 0 & 0 & 1 & 1 & 0 & 1 & 1 \\
4 & 1 & 0 & 0 & 1 & 0 & 0 & 1 \\
5 & 1 & 0 & 1 & 1 & 1 & 0 & 1 \\
6 & 1 & 0 & 1 & 0 & 1 & 0 & 1 \\
7 & 0 & 1 & 0 & 1 & 0 & 1 & 0 \\
8 & 0 & 1 & 1 & 1 & 1 & 1 & 0 \\
9 & 0 & 0 & 1 & 0 & 1 & 1 & 0 \\
10 & 1 & 1 & 1 & 1 & 1 & 0 & 1 \\
11 & 0 & 0 & 1 & 1 & 1 & 1 & 0 \\
\hline
\end{tabular}

\section{Electrical Design}

Decoration of Electrical Design happens based on the equations. The obstacle avoidance circuit diagram consists 10 AND gates, 8 OR gates, 5 NOT gates, 1 Motor driver IC and battery. First IR sensors give the pulse to the gates. Using inverter logic 0 or 1 has been provided to the AND gates. According to the requirements four-input AND gate and fiveinput AND gate have been used. AND gates passes the signals to the $\mathrm{OR}$ gates. Then the logic gates give the commands to motor driver IC. L293D controls motor's speed. A 12 volt battery has been used. The circuit diagram has been shown in Figure 7. 


\section{Mechanical Design}

The implemented obstacle avoidance robot carried out its performance well. The prototype is implemented for educational purpose for university students. The differential drive robot model has been made with a robust design for a hostile environment. The hardware is not jammed permanently; so if damage occurs it can be replaceable.

DDMR is equipped with AND gates, OR gates, Inverters, sensors, a motor driver IC and two de motor. A 12 volt battery gives enough power to run all of these.

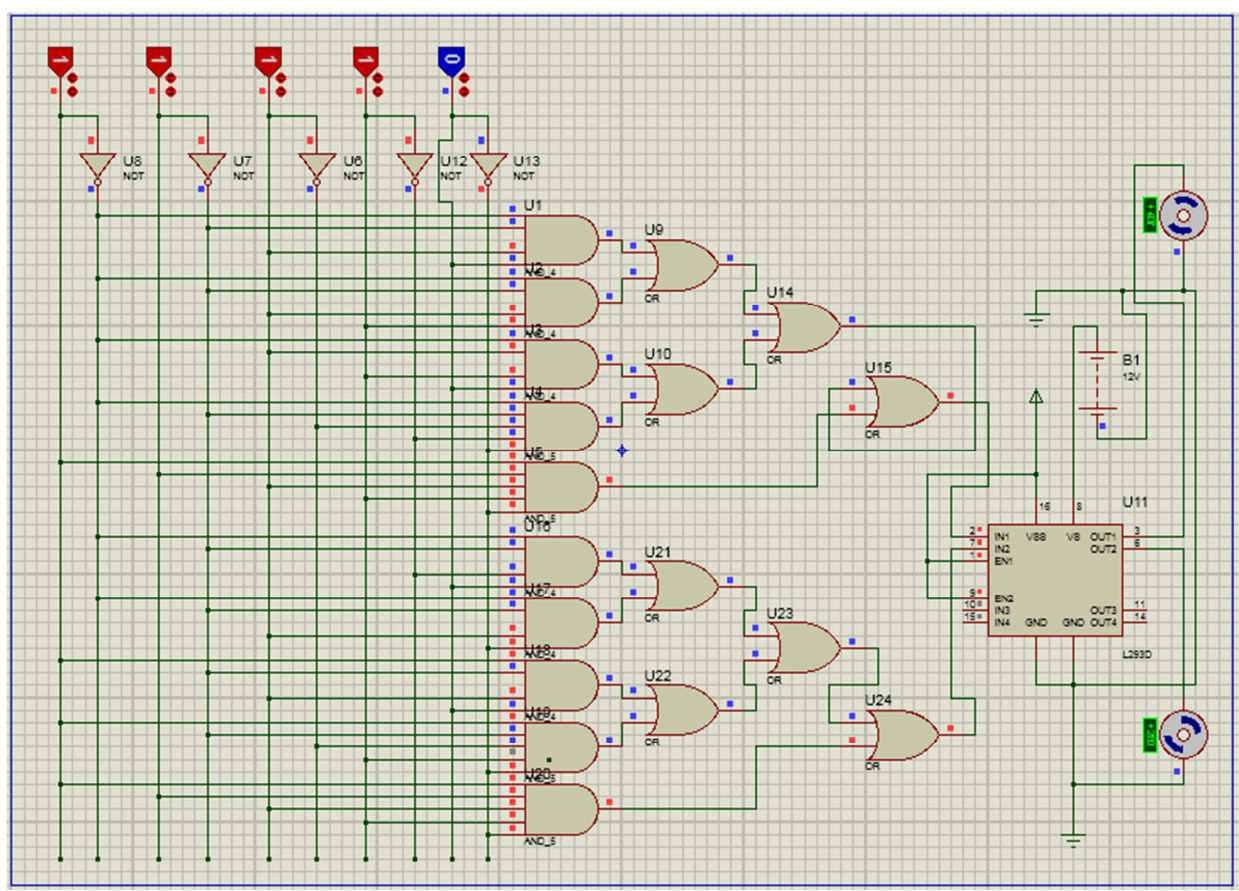

Figure 7. Circuit diagram of DDMR.

One hex inverter IC fulfills the requirement of six inverters. At the same time 5 dual 4-input AND gate, 2 quadruple 2-input AND gate and 2 quadruple 2-input OR gate minimize the circuit and faster the circuit operation. These IC's are very available and the whole design process reducing the cost with respect to the previous version [10]. In indoor situation obstacle avoidance robot has been tested in a path which is approximately 100 inches long and robot passed the test successfully that has been shown in Figure 8 . Measured value for the robot has been given in Table 2 .

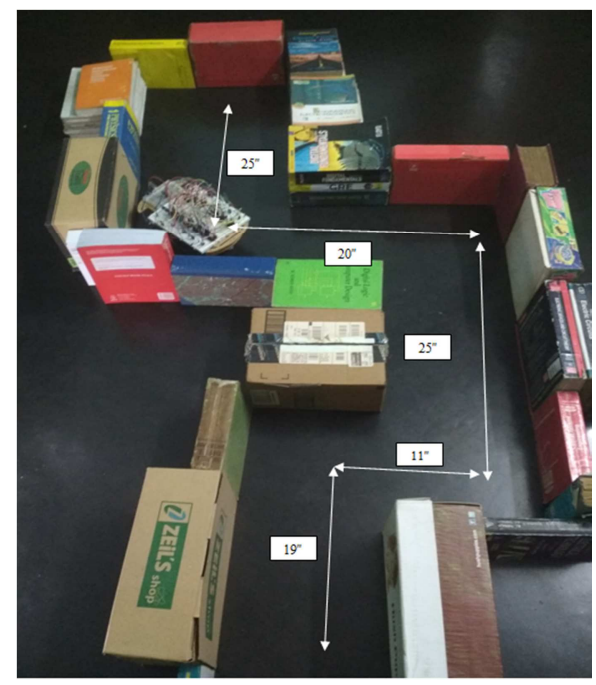

Figure 8. Obstacle Avoidance Robot.
Table 2. Measured Value of the Equipment's.

\begin{tabular}{llll}
\hline Equipments & Length \& Width & Weight & Radius \\
\hline Chassis \& motors & $9.5^{\prime \prime} \& 6 "$ & $186 \mathrm{gm}$ & N/A \\
Upper plate & $10.5^{\prime \prime} \& 6 "$ & $82 \mathrm{gm}$ & N/A \\
Wheel & N/A & $80 \mathrm{gm}$ & $1.25 "$ \\
Battery & N/A & $160 \mathrm{gm}$ & N/A \\
\hline
\end{tabular}

\section{Results \& Discussions}

It has been studied that designed robot model gives more precious direction with respect to previous version [10]. If there are three obstacles in three sides, robot will stop. But for the designed model there are extra sensors to detect obstacles. Suppose left corner sensor detect an obstacle but no obstacle in right corner sensor then robot will move right. Because of these extra sensor robot gets a precious direction. Figure 9 gives a better illustration.

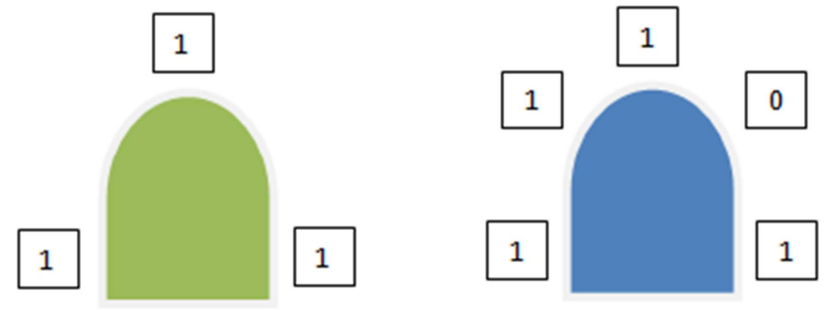

Figure 9. Extra sensors providing precious direction. 
Secondly, more gates are used there is an opportunity to fall the signal strength. That can delay the robot model. By considering these situation gate delay has been calculated for the both of the circuit (previous and new). That is as shown in Figure 6, 7 and 8.

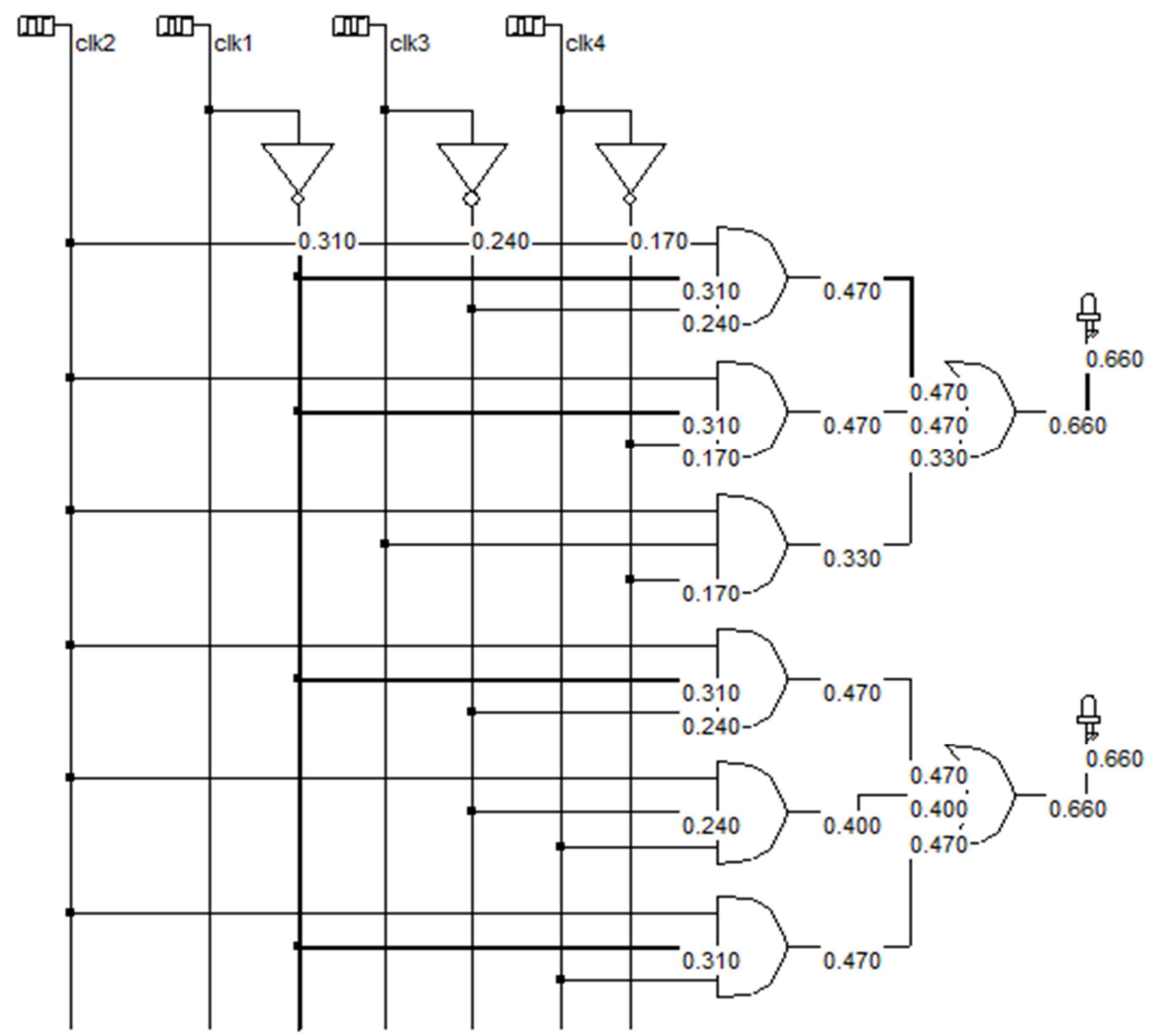

Figure 10. Gate delay of the previous circuit.

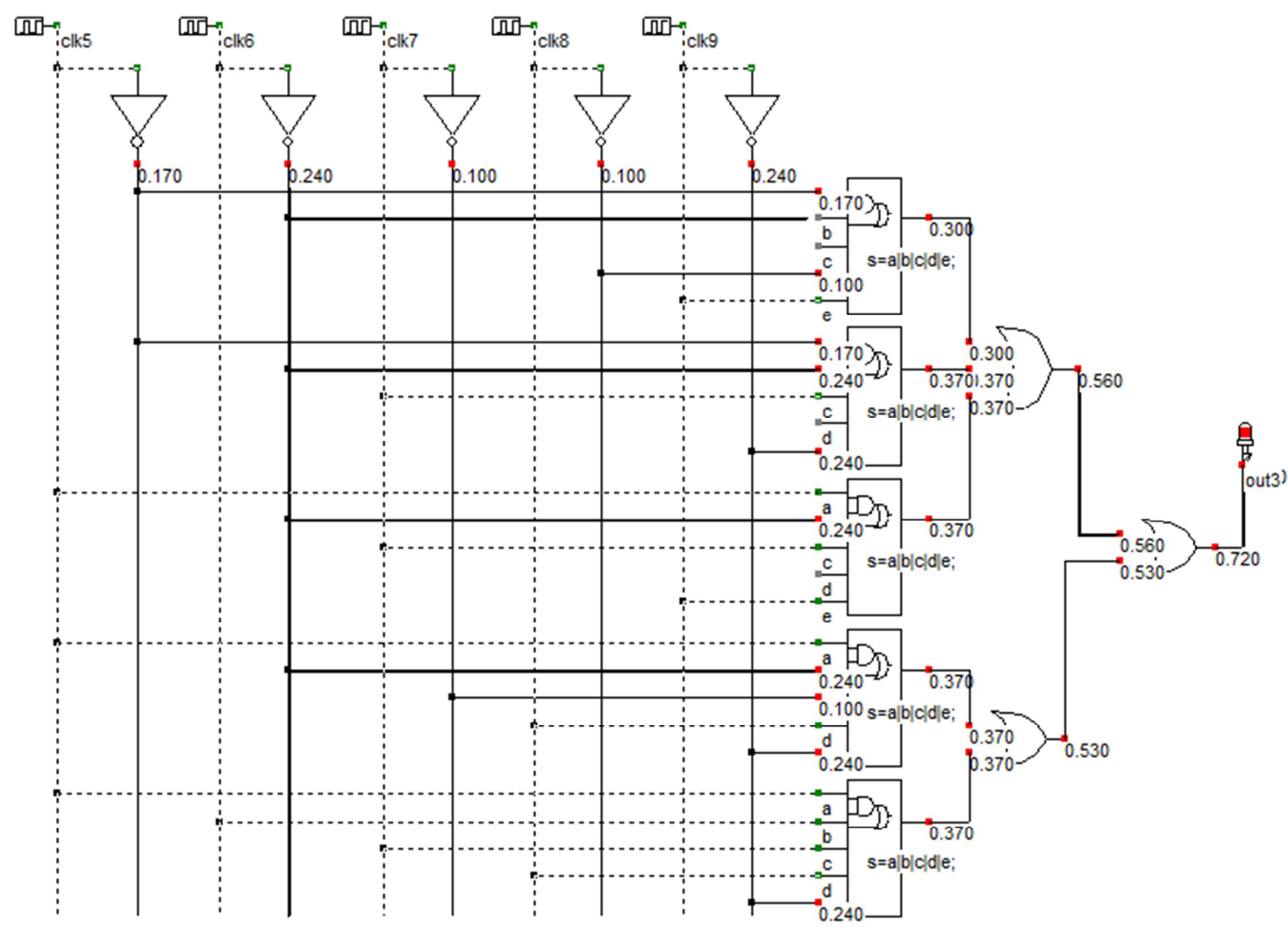

Figure 11. Gate delay of the right motor for implemented circuit. 


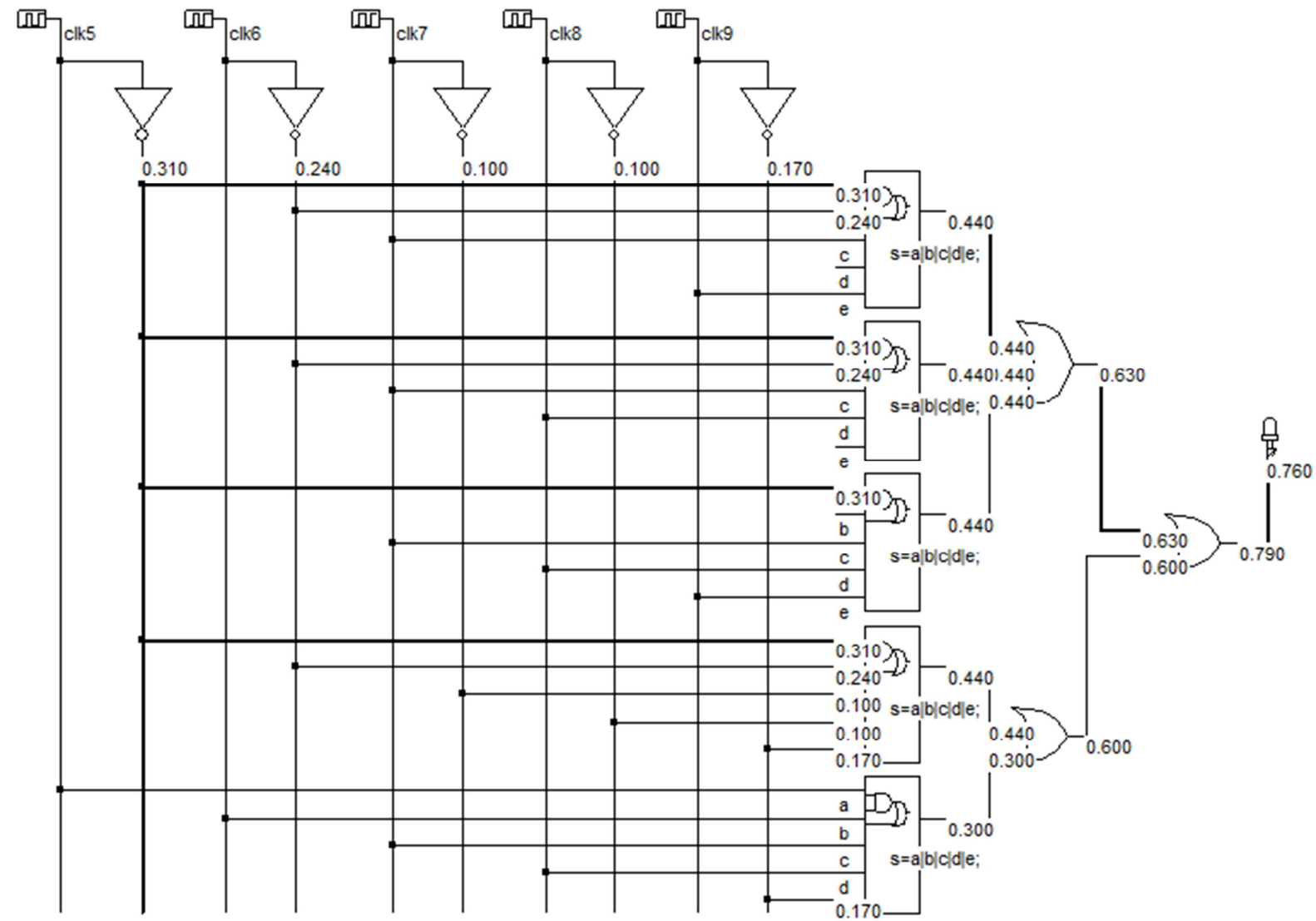

Figure 12. Gate delay of the left motor for implemented circuit.

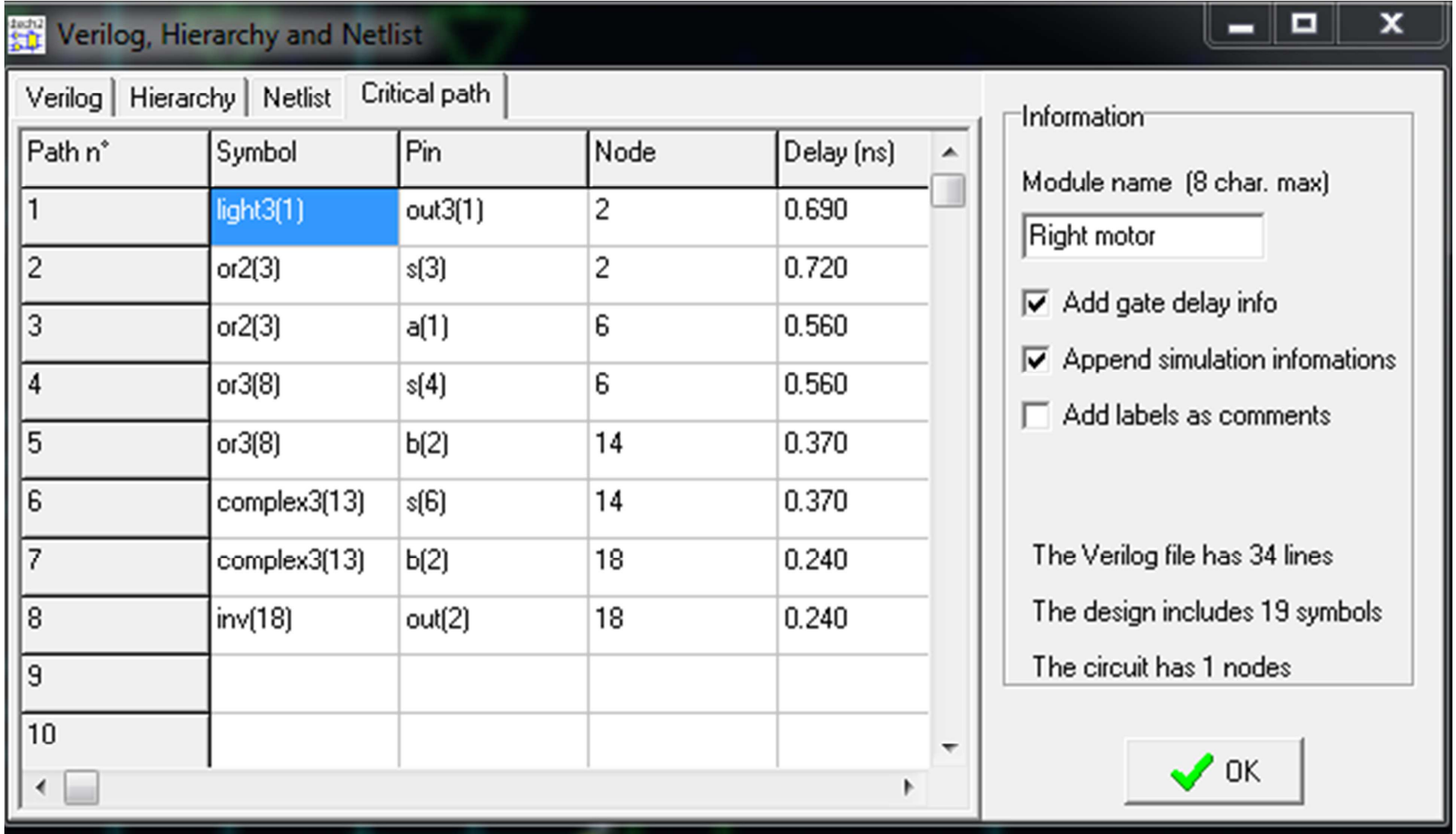

Figure 13. Detail gate delay calculation for implemented right motor. 


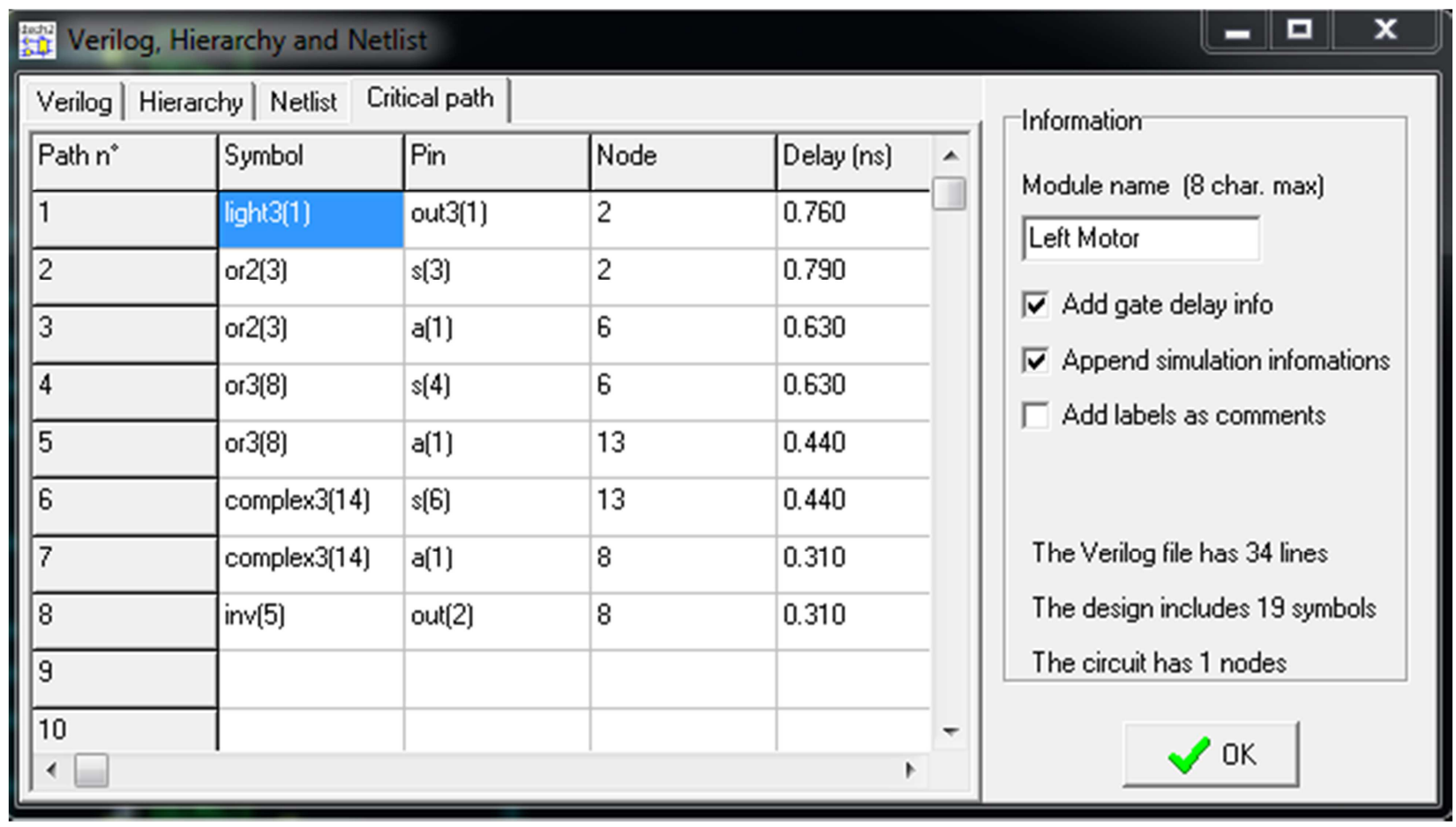

Figure 14. Detail gate delay calculation for implemented left motor.

Figure 10 represent the gate delay for both the right motor and left motor that is $0.66 \mathrm{~ns}$ for the previous circuit [10]. Remarkable point is, for the new modeled robot gate delay for the right motor and left motor is $0.69 \mathrm{~ns}$ and $0.76 \mathrm{~ns}$ respectively. The difference is so little for this type of robot that can be negligible. So, more sensors and gates have been used that increase the accuracy with almost same speed Figure 11 \& Figure 12 shows the designed circuit for right motor and left motor with time delay. Detail delay calculation has been shown in Figure 13 and Figure 14 for designed right motor and left motor respectively.

\section{Conclusions}

This paper presents a detail methodology of a simplest obstacle avoidance robot with time delay calculation with its quondam version. This ramification free design befits every roboticists. Experimental results with simulation demonstrated that the DDMR avoid obstacles within considerable time. The robot has coalesced with various logic gates which is not made it slow moreover gives precious direction. Small ranger IR sensors have been used to conclude this effort in indoor setting. The future work will focus on the path planning with some high quality external and internal sensors.

\section{References}

[1] M. Egeerstedt, Module 2: Mobile robots, Control of mobile robots. Coursera, 2014.
[2] J. Savage, S. Munoz, M. Matamoros, R. Osorio, "Obstacle Avoidance Behaviors for Mobile Robots Using Genetic Algorithms and Recurrent Neural Networks," $6^{\text {th }}$ IFAC Conference onManagement and Control of Production and Logistics, The International Federation of Automatic Control. Fortaleza, Brazil, September 11-13, 2013.

[3] R. C. Arkin, Behaviour-Based Robotics - MIT Press, Cambridge, MA, 1998.

[4] D. M. A. Ahad, A. A. Sarkar, M. A. Mannan, "Mathematical modeling and to carry out a prototype helpmate differential drive robot for hospital purpose," American Academic \& Scholarly Research Journal, vol. 2, no. 6, pp. 1-15, November 2014.

[5] P. Lima and M. I. Ribeiro, "Mobile robotics," Instituto superior teecnico/instituto de sistemas e robotica, March 2002.

[6] H. Henderson, Modern Robotics: Building Versatile Machines, ICBN 0-8160-5745-1, Chelsea House, New York, 2006, pp. 109.

[7] J. Borenstein and Y. Koren, "Real-time obstacle avoidance for fast mobile robots," IEEE Transactions, vol. 19, no. 5, pp. 1179-1187, Sept./Oct. 1989.

[8] D. M. A. Ahad, M. Mynuddin and D. M. Rashid, "Analyzing behaviour of differential drive mobile robot by using statistical tools," J Electr Eng Electron Technol, vol 5: 3, DOI: 10.4172/2325-9833.1000130, 2016.

[9] S. M. Lavalle, "Planning Algorithms," Cambridge University Press, 2006

[10] P. Agrahri, A. Kaur, H. K. Dhanoa and G. Singh, "Obstacle avoiding robot using karnaugh map," International Journal of Applied Engineering Research, ISSN 0973-4562, vol. 10, no. 9, pp. 21983-21990, 2015. 